\title{
Bioimaging of botulinum toxin and hyaluronate hydrogels using zwitterionic near-infrared fluorophores
}

\author{
Ki Su Kim ${ }^{1,2}$, Yun Seop Kim³ ${ }^{3}$ Kai Bao ${ }^{4}$, Hideyuki Wada ${ }^{4}$, Hak Soo Choi ${ }^{4^{*}}$ and Sei Kwang Hahn ${ }^{1,3^{*}}$
}

\begin{abstract}
Background: The injection of botulinum toxin (BTX) to reduce facial wrinkles is one of the most frequently performed plastic surgery procedures. The biocompatible hydrogels are injected with BTX for effective tissue augmentation. However, it is difficult to determine the interval of injection for effective tissue augmentation.
\end{abstract}

Method: BTX and hyaluronate (HA) hydrogels were labeled with zwitterionic (ZW) near-infrared (NIR) fluorophores and visualized for 3 weeks after injection to BALB/c nude mice.

Results: BTX-ZW conjugates and diaminohexane (DAH)-HA-ZW hydrogels were successfully prepared by the conventional EDC/NHS chemistry. Using the NIR fluorescence imaging, we confirmed that approximately $10 \%$ of BTX-ZW conjugates and 50\% of DAH-HA-ZW hydrogels remained 3 weeks post-injection.

Conclusion: This bioimaging technique using invisible NIR fluorescence light can be exploited for various biomedical applications.

Keywords: Hyaluronate, Botulinum toxin, Zwitterionic fluorophore, Tissue augmentation, Bioimaging

\section{Background}

Botulinum toxin (BTX), a neurotoxic protein derived from the bacterium Clostridium botulinum, is most frequently used for removing facial wrinkles [1, 2]. Small quantities of BTX can cause relaxation of overactive muscles and reduce wrinkles by smoothing overlying skin [3]. BTX inhibits acetylcholine release and causes temporary chemical denervation at the neuromuscular junction by cleaving the synaptosomal-associated protein of $25 \mathrm{kDa}$ [SNAP-25] on the internal surface of neuronal membranes, followed by vesicle fusion at the cellular level [4]. After SNAP-25 regenerates over time, BTX effects diminish in the targeted muscles, and neuromuscular signaling and muscle contractility are restored [4].

Biocompatible hydrogels are generally injected with BTX to help fill skin wrinkles and effectively augment

\footnotetext{
* Correspondence: hchoi12@mgh.harvard.edu; skhanb@postech.ac.kr

${ }^{4}$ Gordon Center for Medical Imaging, Department of Radiology,

Massachusetts General Hospital and Harvard Medical School, 149 13th Street, Boston, MA 02129, USA

'PHI BIOMED Co., \#613, 12 Gangnam-daero 65-gil, Seocho-gu, Seoul 06612 ,

South Korea

Full list of author information is available at the end of the article
}

tissue volume [5, 6]. Among injectable dermal fillers, hyaluronate (HA) hydrogels have become especially popular for soft tissue augmentation since HA can absorb water to recover the volume of aging tissue [7-10]. HA fillers with a larger particle size and higher molecular weight are generally preferred to extend the duration under the skin, which can be obtained by crosslinking HA [11-13]. The typical dose of BTX is 200 unit in 3 months interval [14]. The use of BTX and dermal fillers, however, threatens healthcare workers and patients due to residue left in the body, and it is a challenge to determine the dosing interval for effective tissue augmentation. Moreover, there have been previously reported the efficacy of Botox through bioimaging techniques $[14,15]$. However, there were no studies to confirm the in vivo behavior of BTX.

Here, we firstly investigated in vivo dynamics of BTX and dermal fillers for tissue augmentation using nearinfrared (NIR) fluorescence, which penetrates deeply into biological tissues [16]. We previously reported that zwitterionic (ZW) NIR fluorophores have low serum binding, low nonspecific tissue uptake, and rapid elimination from the body through renal filtration [17]. ZW 
fluorophores also have superior optical properties (i.e., high extinction coefficient and quantum yield) compared to visible dyes, which together improves quantitative bioimaging [18-21]. In this study, we investigate the in vivo dynamics of BTX and HA hydrogels for tissue augmentation by introducing $\mathrm{ZW}$ fluorophores in the chain.

\section{Methods}

\section{Materials}

Hyaluronate (HA) was purchased from Lifecore Co. (Chaska, MN). Botulinum toxins (BTX, Meditox, Ochang, Korea) were kindly gifted from Dr. Jeesoo An at the Wellman Center for Photomedicine in Massachusetts General Hospital. 1-Ethyl-3-(3-dimethylaminopropyl) carbodiimide (EDC) was obtained from Tokyo Chemical Industry (Tokyo, Japan). N-hydroxysulfosuccinimide (sulfo-NHS), phosphate buffered saline (PBS), diaminohexane (DAH) and hydroxylbenzotriazole (HOBt) were purchased from Sigma (St. Louis, MO). Dialysis membrane tube was obtained from Thermo Scientific Co. (Waltham, MA).

\section{Synthesis of Zwitterionic (ZW) NIR fluorophores}

ZW NIR fluorophores were prepared as reported previously [18]. In brief, Vilsmeier-Haack reagent was used for the condensation reaction with prepared intermediate indolium salts in anhydrous sodium acetate to prepare indocyanine based chloro-subtitued NIR fluorophore. And then, using microwave synthesis, a bifunctional phenoxypropionic acid linkage was introduced on the meso-chlorine atom to permit conjugation of targeting ligands. The crude product was washed against diethyl ether and precipitated in methanol and diethyl ether $(20 \mathrm{~mL}, 1: 4)$ to give the ZW NIR fluorophores.

\section{Labeling of ZW fluorophores}

HA and BTX were labeled with the amine modified ZW fluorophore, with emission wavelengths of $700 \mathrm{~nm}$ and $800 \mathrm{~nm}$, respectively. HA (MW $100 \mathrm{kDa}$ ) was dissolved at a concentration of $5 \mathrm{mg} / \mathrm{mL}$ in double distilled water. After complete dissolution, ZW fluorophores (1 M ratio of $\mathrm{HA}$ ) were added to the HA solution, and EDC and sulfo-NHS were added with $4 \mathrm{M}$ ratio of $\mathrm{HA}$ to activate the carboxyl groups of HA. The $\mathrm{pH}$ of the reaction mixture was maintained at 6.5 , and ZW fluorophore (1 M ratio of HA) was added to the solution and stirred overnight. After the reaction was stopped by changing the $\mathrm{pH}$ to 7.4 , the resulting $\mathrm{HA}-\mathrm{ZW}$ conjugate was purified with gel permeation chromatography (GPC) measuring the retention time. The mobile phase was $\mathrm{PBS}$ at $\mathrm{pH} 7.4$ and the flow rate was $1 \mathrm{~mL} / \mathrm{min}$. The detection wavelength was $210 \mathrm{~nm}$. The purified conjugate solution was lyophilized for 3 days. BTX was dissolved in phosphate buffered saline (PBS, Sigma, St. Louis, MO) and the conjugation and purification was performed as described above. The detection wavelength was $280 \mathrm{~nm}$.

\section{Preparation of DAH-HA-ZW hydrogels}

DAH-HA-ZW hydrogel was prepared using the same method described elsewhere [22]. HA-ZW conjugates were dissolved at a concentration of $30 \mathrm{mg} / \mathrm{mL}$ in double distilled water; after complete dissolution, DAH ( $1 \mathrm{M}$ ratio of $\mathrm{HA}$ ) was added to the HA solution for a cross-linking reaction with the carboxyl groups of HA. EDC and HOBt ( $1 \mathrm{M}$ ratio of $\mathrm{HA}$ ), activating the carboxyl groups of HA, were dissolved in water and added to the mixed solution of HA and DAH for DAH-HA hydrogel preparation. The final precursor solution was incubated at $37{ }^{\circ} \mathrm{C}$ for $2 \mathrm{~h}$ to complete the cross-linking reaction. Prepared DAH-HA-ZW hydrogels were sealed within dialysis membrane tube (MWCO of $7 \mathrm{kDa}$ ) and dialyzed against PBS for 3 days to remove the remaining EDC, HOBt, and DAH completely. The degree of modification in DAH-HA conjugates was analyzed by proton nuclear magnetic resonance $\left({ }^{1} \mathrm{H}\right.$ NMR, DRX-400, Bruker, Germany).

\section{Bioimaging of BTX-ZW conjugates and DAH-HA-ZW hydrogels}

BTX-ZW conjugates and DAH-HA-ZW hydrogel with the same fluorescence intensity were intramuscularly and subcutaneously inoculated into the BALB/c nude mice (100 pmol of ZW dye, $50 \mu \mathrm{L}$ ), sequentially. The home-built dual-channel imaging system [21] was used to acquire NIR fluorescent images at $0,1,2$, and 3 weeks post-injection. Animals were housed in an AAALACcertified facility and all animal studies were performed under the supervision of BIDMC IACUC in accordance with approved institutional protocol of \#057-2014.

\section{Results and discussion Synthesis of BTX-ZW conjugates and DAH-HA-ZW hydrogel}

Figure 1 shows the schematic representations for the chemical synthesis of BTX conjugates and HA hydrogel with ZW fluorophores. The ZW fluorophores emit NIR wavelength and avoid nonspecific tissue uptake and serum protein association due to their net charge. In plastic surgery, BTXs were typically injected in muscle, which is a deeper site than the subcutaneous injection of hydrogels for tissue augmentation. The carboxyl group of BTX was modified with amine-modified ZW800-1 fluorophore, which has a longer wavelength of fluorescence for intramuscular injection. In addition, HA was conjugated with ZW700-1, which has a shorter wavelength. The ZW labeled HA hydrogel was formed by the crosslinking of DAH. Since the amine groups of unreacted DAH crosslinkers were reported to be 
a

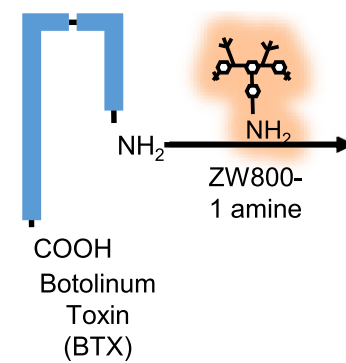

C
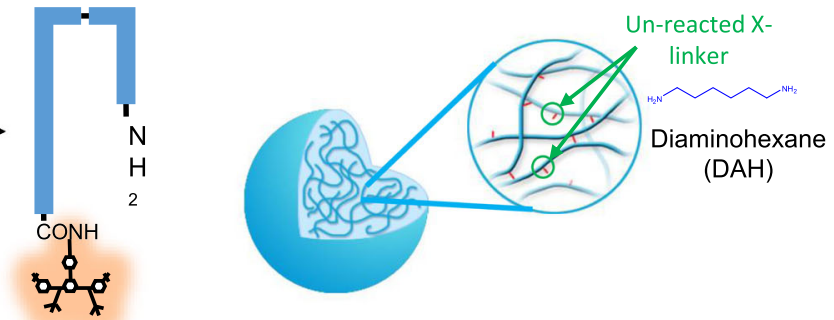

BTX-ZW800-1

b

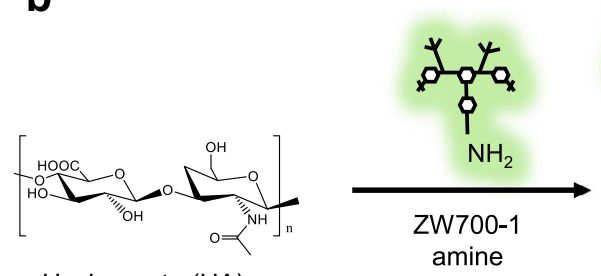

Hyaluronate (HA)

amine

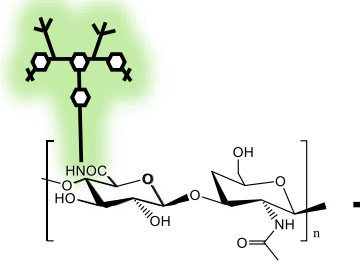

HA-ZW700-1

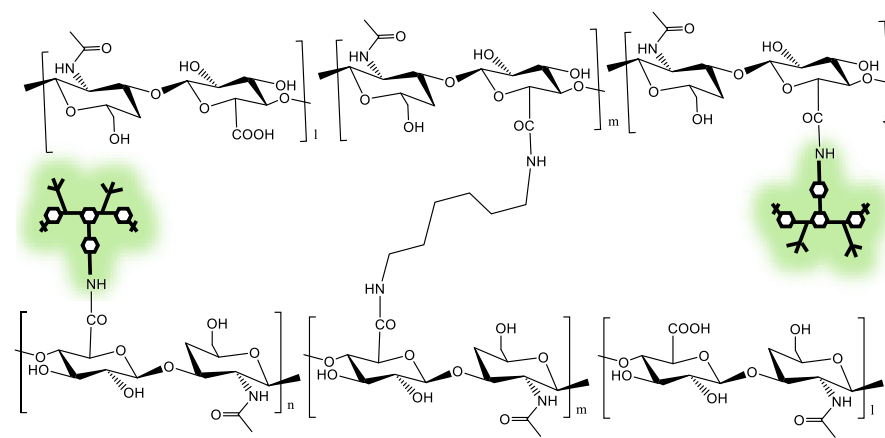

ZW700-1-HA-DAH Hydrogel

Fig. 1 Schematic diagrams for the synthesis of (a) BTX-ZW conjugates and (b) DAH-HA-ZW hydrogel, and for the illustrated structure of (c) DAH-HA hydrogel

nontoxic and promote the attachment and proliferation of keratinocytes and fibroblasts [23], DAH-HA-ZW hydrogel can be exploited for tissue augmentation.

\section{Characterization of the BTX-ZW, HA-ZW, and DAH-HA conjugates}

The resulting BTX-ZW and HA-ZW conjugates were characterized and purified by GPC analysis (Fig. 2a). The retention time of native BTX-ZW with a MW of $150 \mathrm{kDa}$ was ca. $3 \mathrm{~min}$, and the retention time of HAZW with a MW of $100 \mathrm{kDa}$ was ca. $1.5 \mathrm{~min}$. The retention time of the mixture of HA-ZW and BTX-ZW was ca. 1.5 min. Successful conjugation of DAH to HA was confirmed by ${ }^{1} \mathrm{H}$ NMR analysis (Fig. 2b). The pattern of ${ }^{1} \mathrm{H}$ NMR spectrum of DAH-HA conjugates was identical with our previous work [24], and the integral ratio on the ${ }^{1} \mathrm{H}$ NMR spectrum suggested that, on average, 18 mol\% of HA units was modified with DAH. The methyl resonance $(\delta=1.85-2.05 \mathrm{ppm})$ of acetamido moiety of $\mathrm{N}$-acetyl-D-glucosamine residue in $\mathrm{HA}$ was used as an internal standard, and the degree of DAH-HA modification was determined from the peak area of methylenes of DAH at $\delta=1.3 \mathrm{ppm}$.

\section{Dynamics of BTX-ZW conjugates and DAH-HA-ZW hydrogel}

Based on characterization, we investigated the dynamics of BTX and HA hydrogel in vivo. The ZW labeled BTX and HA hydrogel were administered with intramuscular and subcutaneous injection, respectively. NIR fluorescent images were acquired with emission wavelength of $700 \mathrm{~nm}$ (for ZW700-1) and $800 \mathrm{~nm}$ (for ZW800-1) by fluorescence-assisted resection and exploration (FLARE) imaging. As control, ZW800-1 with intramuscular injection and ZW700-1 with subcutaneous injection were also tested for comparison. In Fig. 3, almost no fluorescence signal of control group remained at injection site within 1 week. Based on previous work [20], the control 

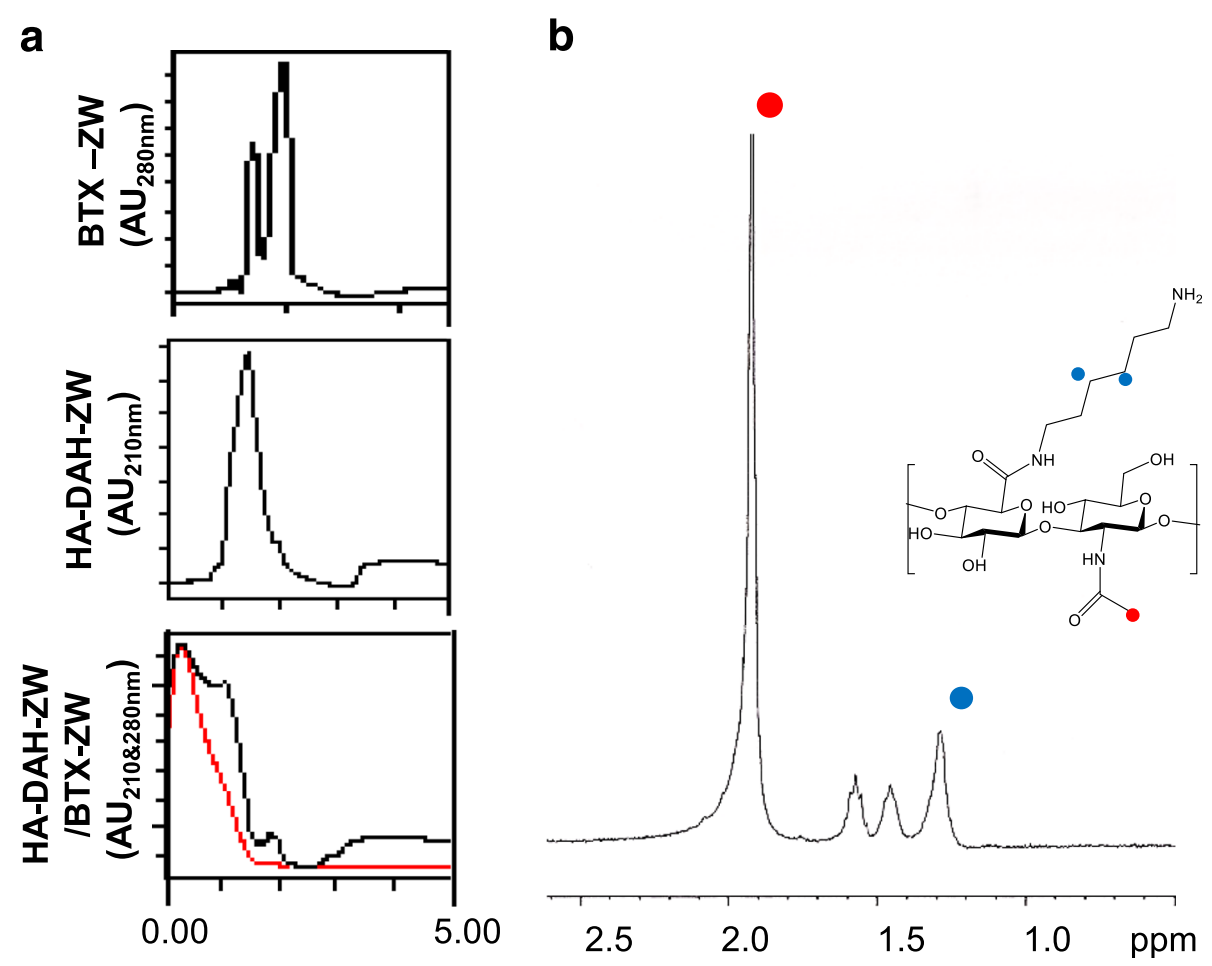

Fig. 2 a The gel-permeation chromatogram of BTX, HA, and the mixture of HA and BTX. $\mathbf{b}$ NMR spectrum of DAH-HA conjugates

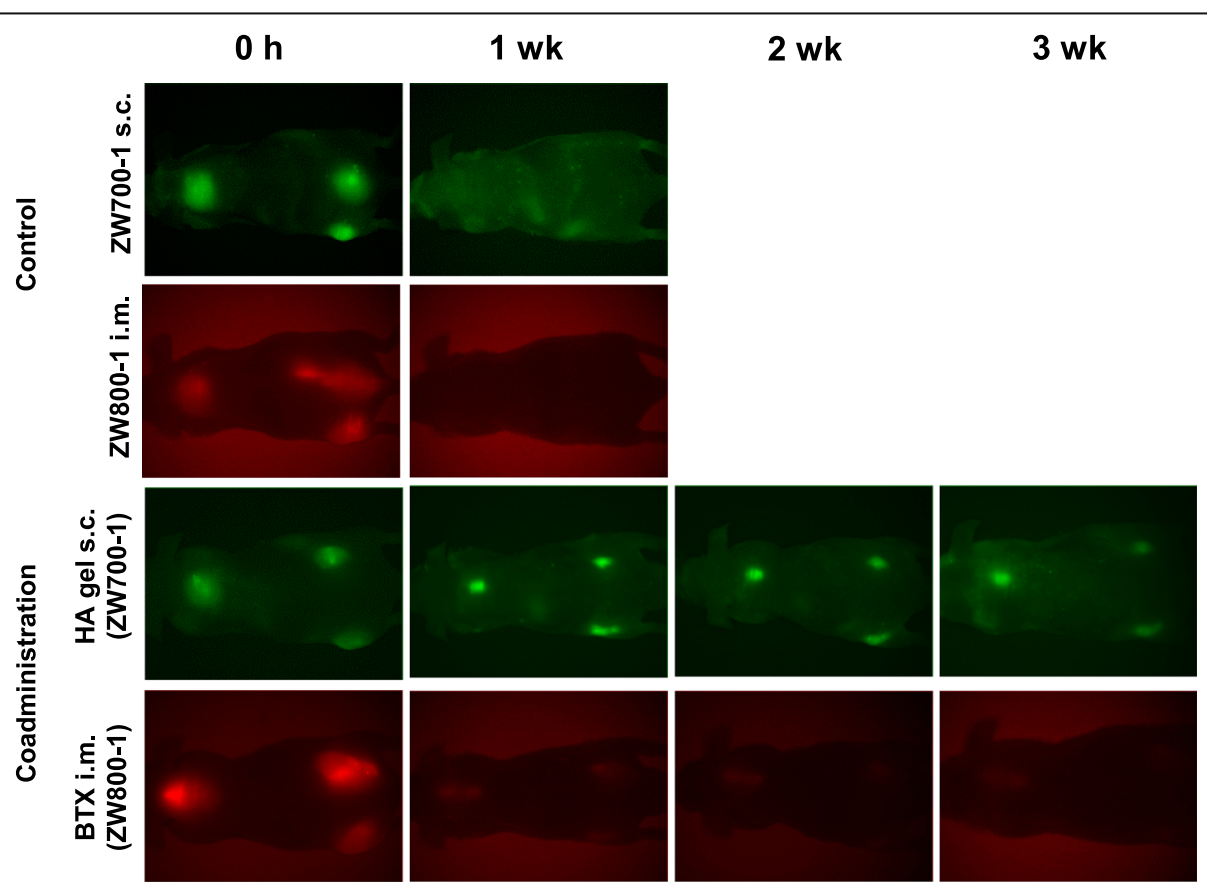

Fig. 3 NIR fluorescence images of ZW700-1 with subcutaneous (s.c.) injection, ZW800-1 with intramuscular (i.m.) injection, DAH-HA-ZW hydrogel with s.c. injection, and BTX-ZW conjugates with i.m. injection, in order from top to bottom 


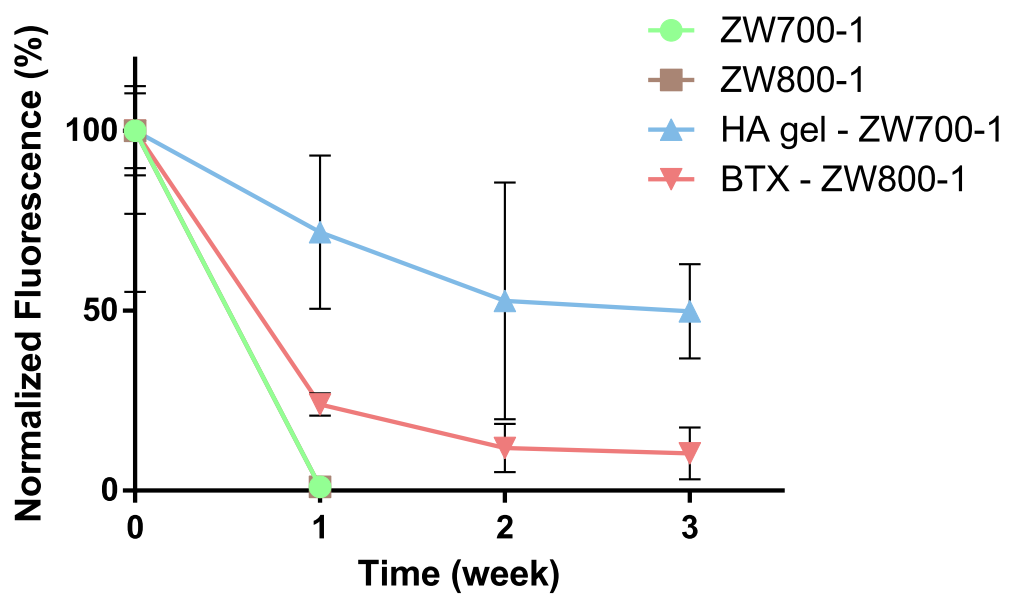

Fig. 4 Quantification of fluorescence intensity after i.m injections of ZW800-1 and BTX-ZW conjugates, s.c. injections of ZW700-1 and DAH-HA-ZW hydrogel

group may also clear within 1 day from the body. BTXZW conjugates and DAH-HA-ZW hydrogel remained in the body for over 3 weeks, but the remaining pattern of BTX-ZW conjugates differed from the DAH-HA-ZW hydrogel pattern. The BTX-ZW conjugates cleared quickly, caused by enzymatic degradation. However, since the crosslinked HA can avoid enzymatic degradation, DAH-HA-ZW hydrogel could remain for over 3 weeks.

Based on FLARE images, the in vivo dynamics of fluorescence was measured by quantifying fluorescence injection of the injection site (Fig. 4). Since all samples with ZW fluorophore were inoculated by injection, we normalized the fluorescence intensity to $100 \%$ at 0 week. Both BTX and DHA-HA hydrogel remained in the body longer when compared to the control group. Over 50\% HA hydrogel remained subcutaneously, while $10 \%$ of BTX remained in muscle after 3 weeks of administration. BTX was easily removed due to its small size and enzymatic degradation. Overall, we could confirm the amount and body residue of BTX and HA hydrogels using bioimaging with ZW fluorophore. This strategy for indicating body residue and dosing interval of agents can be harnessed for various biomedical applications.

\section{Conclusions}

Bioimaging techniques using Zwitterionic (ZW) NIR fluorophores were successfully carried out to investigate in vivo dynamics of each component for tissue augmentation. BTX-ZW conjugates and DAH-HA-ZW hydrogel were synthesized, and in vivo dynamics were investigated using home-built bioimaging equipment. Bioimaging using $\mathrm{ZW}$ fluorophores for indication of bodily residue and the interval of agents can be exploited for various biomedical applications.
Acknowledgements

We thank Dr. Wonjin Park (W Beauty Medical Group) for many helpful discussion. We also thank Ivey Choi for manuscript editing and Eric McDonald for supporting animal study.

\section{Funding}

This research was supported by the US National Institutes of Health grant NIBIB \#R01-EB-022230. This study was also supported by the Bio \& Medical Technology Development Program (No. 2012M3A9C6049791), Mid-career Researcher Program (No. 2015R1A2A1A15053779) of the National Research Foundation (NRF) funded by the Ministry of Science, ICT \& Future Planning, Korea

Availability of data and materials

Please contact author for data request.

\section{Authors' contributions}

KSK mainly designed and performed all experiments, collected samples, analyzed and interpreted data, prepared the figures for the manuscript, and wrote the manuscript. SKH and HSC conceived and supervised the project, designed experiments, interpreted data and wrote the manuscript. YSK contributed to the chemical synthesis. KB and HW contributed to preparing and designing the animal experiments. All authors read and approved the final manuscript.

Ethics approval and consent to participate Not applicable.

\section{Consent for publication}

The manuscript has been submitted with the consent of all authors for publication.

\section{Competing interests}

The authors declare that they have no competing interests.

\section{Publisher's Note}

Springer Nature remains neutral with regard to jurisdictional claims in published maps and institutional affiliations.

\section{Author details}

'PHI BIOMED Co., \#613, 12 Gangnam-daero 65-gil, Seocho-gu, Seoul 06612 , South Korea. ${ }^{2}$ Department of Organic Materials Science and Engineering, College of Engineering, Pusan National University, 2 Busandaehak-ro 63 beon-gil, Geumjeong-gu, Busan 46241, South Korea. ${ }^{3}$ Department of Materials Science and Engineering, Pohang University of Science and 
Technology (POSTECH), 77 Cheongam-ro, Nam-gu, Pohang, Gyeongbuk 37673, South Korea. ${ }^{4}$ Gordon Center for Medical Imaging, Department of Radiology, Massachusetts General Hospital and Harvard Medical School, 149 13th Street, Boston, MA 02129, USA.

Received: 18 July 2017 Accepted: 11 September 2017 Published online: 10 October 2017

\section{References}

1. Small R. Botulinum toxin injection for facial wrinkles. Am Fam Physician. 2014;90:168-75.

2. Montecucco C, Molgó J. Botulinal neurotoxins: revival of an old killer. Curr Opin Pharmacol. 2015:5:274-9.

3. Dressler D, Benecke R. Pharmacology of therapeutic botulinum toxin preparations. Disabil Rehabil. 2007:29:1761-8.

4. Peng L, Liu H, Ruan $H$, Tepp WH, Stoothoff $W H$, Brown RH, Johnson EA, Yao WD, Zhang SC, Dong M. Cytotoxicity of botulinum neurotoxins reveals a direct role of syntaxin 1 and SNAP-25 in neuron survival. Nat Commun. 2013:4:1472.

5. Coleman KR, Carruthers J. Combination therapy with BOTOX ${ }^{\mathrm{TM}}$ and fillers: the new rejuvenation paradigm. Dermatol Ther. 2006;19:177-88.

6. Carruthers J, Carruthers A. A prospective, randomized, parallel group study analyzing the effect of BTX-A (Botox) and nonanimal sourced hyaluronic acid (NASHA, Restylane) alone in combination compared with NASHA (Restylane) alone in severe glabellar rhytides in adult female subjects: treatment of severe glabellar rhytides with a hyaluronic acid derivative compared with the derivative and BTX-A. Dermatol Surg. 2003;29:802-9.

7. Monheit GD, Coleman KM. Hyaluronic acid fillers. Dermatol Ther. 2006;19:141-50.

8. Cohen JL. Understanding, avoiding, and managing dermal filler complications. Dermatol Surg. 2008;34:S92-9.

9. Kablik J, Monheit GD, Yu L, Chang G, Gershkovich J. Comparative physical properties of hyaluronic acid dermal fillers. Dermatol Surg. 2009;35:302-12.

10. Klein AW, Elson ML. The history of substances for soft tissue augmentation. Dermatol Surg. 2000;26:1096-105.

11. Sparavigna A, Fino P, Tenconi B, Giordan N, Amorosi V, Scuderi N. A new dermal filler made of cross-linked and auto-cross-linked hyalurnoic acid in the correction of facial aging defects. J Cosmet Dermatol. 2014;13:307-14.

12. Yeom JS, Hwang BW, Yang DJ, Shin HI, Hahn SK. Effect of osteoconductive hyaluronate hydrogels on calvarial bone regeneration. Biomater Res. 2014;18:8.

13. Chun C, Lee DY, Kim JT, Kwon MK, Kim YZ, Kim SS. Effect of molecular weight of hyaluronic acid $(\mathrm{HA})$ on viscoelasticity and particle texturing feel of HA dermal biphasic fillers. Biomater. Res. 2016;20:24.

14. Wollina U, Konrad H, Petersen S. Botulinum toxin in dermatology - beyond wrinkles and sweat. J Cosmet Dermatol. 2005:4:223-7.

15. Friedman PM, Skover GR, Payonk G, Kauvar ANB, Geronemus RG. 3D in-vivo optical skin imaging for topographical quantitative assessment of nonablative laser technology. Dermatol Surgery. 2002;28:199-204.

16. Jacobi U, Chen M, Frankowski G, Sinkgraven R, Hund M, Rzany B, Sterry W, Lademann J. In vivo determination of skin surface topography using an optical 3D device. Skin Res Technol. 2004;10:207-14.

17. Zaheer A, Lenkinski RE, Mahmood A, Jones AG, Cantley LC, Frangioni JV. In vivo near-infrared fluorescence imaging of osteoblastic activity. Nat Biotechnol. 2001;19:1148-54

18. Choi HS, Nasr K, Alyabyev S, Feith D, Lee JH, Kim SH, Ashitate Y, Hyun H, Patonay G, Strekowski L, Henary M, Frangioni JV. Synthesis and in vivo fate of zwitterionic near-infrared fluorophores. Angew Chem Int Ed. 2011;50:6258-63.

19. Choi HS, Gibbs SL, Lee JH, Kim SH, Ashitate Y, Liu F, Hyun H, Park GL, Xie Y, Bae S, Henary M, Frangioni JV. Targeted zwitterionic near-infrared fluorophores for improved optical imaging. Nat Biotechnol. 2013;31:148-53.

20. Kim KS, Hyun H, Yang JA, Lee MY, Kim H, Yun SH, Choi HS, Hahn SK. Bioimaging of hyaluronate-interferon a conjugate using non-interfering zwitterionic fluorophore. Biomacromolecules. 2015;16:3054-61.

21. Hyun H, Wada H, Bao K, Gravier J, Yadav Y, Laramie M, Henary M, Frangioni JV, Choi HS. Phosphonated near-infrared fluorophores for biomedical imaging of bone. Angew Chem Int Ed. 2014;53:10668-72.

22. Yeom J, Bhang SH, Kim BS, Seo MS, Hwang EJ, Cho IH, Park JK, Hahn SK. Effect of cross-linking reagents for hyaluronic acid hydrogel dermal fillers on tissue augmentation and regeneration. Bioconjug Chem. 2010;21:240-7.
23. Mckegney M, Taggart I, Grant M. The influence of crosslinking agents and diamines on the pore size, morphology and the biological stability of collagen sponges and their effect on cell penetration through the sponge matrix. J Mater Sci Mater Med. 2001;12:833-44.

24. Park KM, Yang JA, Jung H, Yeom J, Park JS, Park KH, Hoffman AS, Hahn SK, Kim K. In situ supramolecular assembly and modular modification of hyaluronic acid hydrogels for 3D cellular engineering. ACS Nano. 2012;6:2960-8.

\section{Submit your next manuscript to BioMed Central and we will help you at every step:}

- We accept pre-submission inquiries

- Our selector tool helps you to find the most relevant journal

- We provide round the clock customer support

- Convenient online submission

- Thorough peer review

- Inclusion in PubMed and all major indexing services

- Maximum visibility for your research

Submit your manuscript at www.biomedcentral.com/submit
) Biomed Central 